\title{
Archives
}

\section{La mémoire utile, fond et forme au menu des années $\mathbf{2 0 0 0}$}

\section{CLAUDE MINOTTO}

Volume 46, numéro 2, 2017

\section{$50^{\mathrm{e}}$ anniversaire de la DGDA}

URI : https://id.erudit.org/iderudit/1040378ar

DOI : https://doi.org/10.7202/1040378ar

Aller au sommaire du numéro

\section{Éditeur(s)}

Association des archivistes du Québec (AAQ)

ISSN

0044-9423 (imprimé)

2369-9256 (numérique)

Découvrir la revue

Citer cet article

Minotto, C. (2017). La mémoire utile, fond et forme au menu des années 2000. Archives, 46(2), 27-33. https://doi.org/10.7202/1040378ar

\section{Résumé de l'article}

À l'occasion du $50^{\mathrm{e}}$ anniversaire de la Division de la gestion de documents et des archives de l'Université de Montréal, Claude Minotto, son ancien directeur nous livre ses réflexions. Il nous présente d'abord un bref plaidoyer en faveur de la diversité, car à ses yeux, une fois les conditions de préservation assurées, l'important est l'utilité de ce qui est conservé. Il utilise l'expression qui sert d'entête au site Internet de la DGDA comme préambule à la présentation de divers défis qu'ont amené les changements technologiques de la première décennie des années 2000 à la discipline archivistique. Monsieur Minotto partage ensuite quelques anecdotes issues de ses années en tant que directeur de la DGDA afin d'illustrer l'importance des relations de cette dernière avec les autres entités de l'Université de Montréal. Il termine en soulignant différentes réalisations de l'équipe de la DGDA au cours de sa direction et confirme, en conclusion, la nécessité de préserver « la mémoire utile » et ce, peu importe le support et les évolutions technologiques.
Tous droits réservés @ Association des archivistes du Québec (AAQ), 2017
Ce document est protégé par la loi sur le droit d'auteur. L’utilisation des services d'Érudit (y compris la reproduction) est assujettie à sa politique d'utilisation que vous pouvez consulter en ligne.

https://apropos.erudit.org/fr/usagers/politique-dutilisation/ 


\section{ANNIVERSAIRE DE LA DGDA}

\section{La mémoire utile, fond et forme au menu des années 2000}

\section{CLAUDE MINOTTO}

Ancien directeur, Division de la gestion de documents et des archives, Université de Montréal

\section{INTRODUCTION}

À l'invitation de Diane Baillargeon, directrice de la Division de la gestion de documents et des archives (DGDA), trois anciens directeurs vous auront fait part de leur expérience à ce titre. Avant de commencer ma présentation, je voudrais souligner le courage et le talent de Diane pour convier et contrôler autant de «belles-mères » ou plutôt, en l'occurrence, de «beaux-pères »!

\section{PAPIER VS NUMÉRIQUE: LE NATUREL, LE SYNTHÉTIQUE, L'AUTHENTIQUE... LA RÉALITÉ}

La dichotomie de l'analogique vs le numérique, plus souvent nommée "papier vs numérique», a réellement marqué les archives au tournant du millénaire. Pour illustrer ce rapport entre les supports, j'aimerais vous soumettre à un petit exercice d'analogie concernant le naturel et le 
synthétique. Veuillez lever la main bien haut toutes celles et ceux qui, dans leurs vêtements, n'ont aucune fibre ou pièce que l'on dit synthétique, c'est-à-dire de l'acrylique, polyester, nylon, rayonne, polyuréthane ou autre... Maintenant, levez la main ceux dont les vêtements ne contiennent aucune fibre ou pièce dite naturelle, telle que le coton, la laine, le cuir ou autre... La réalité, c'est que tous, vous portez et vous vous présentez dans une diversité et une mixité de tissus, et vous n'en êtes pas moins des personnes intègres ou authentiques. La diversité existe aussi dans les institutions et les archives institutionnelles. Bien sûr pour les documents à plus longue durée utile, voire les archives permanentes, il y a des conditions de durabilité et de pérennité. Les normes ISO pour le papier comme pour les formats numériques sont là pour être prises en compte. Car ce qui compte le plus, c'est l'utilité, c'est la mémoire utile.

\section{MAIS QU'EST-CE QUE LA « MÉMOIRE UTILE » ?}

La mémoire utile, c'est tout simplement la mémoire qui vous apporte en temps opportun l'information et l'inspiration nécessaires. Et mes efforts à cet égard, pendant une décennie à la DGDA, peuvent se résumer en une seule phrase de moins de 10 mots: "Pour une mémoire institutionnelle d'utilité courante et historique». Cette phrase coiffe encore la page d'accueil du site internet de la DGDA. Cette phrase est un élan, une devise, en quelque sorte, qui n'est pas de moi seul, et j'en suis d'autant plus fier. Elle résulte d'un remue-méninges bref et spontané dans le local de Michel Champagne, devant la maquette de la première refonte du site internet. C'est une phrase qui reflète une communauté de vues et une collaboration exemplaire. Une phrase qui résume en quelques mots la mission aujourd'hui cinquantenaire de la DGDA.

\section{BIEN DES CHANGEMENTS À L'APPROCHE DES ANNÉES 2000}

La production des documents s'est complètement métamorphosée à I'approche et au début des années 2000. Un nouvel univers documentaire s'est créé avec la combinaison et l'effet conjoint de l'internet, des suites bureautiques (avec texteurs, chiffriers, logiciels graphiques et de présentations), du courriel et des fichiers joints.

Des chantiers et des ateliers de numérisation massive ont été mis en place dans plusieurs types d'organismes. En 1998, les Archives nationales 
du Québec (alors ANQ) ont produit le Guide d'imagerie numérique, rejoignant une foule d'utilisateurs publics et privés, à l'intention première des organismes publics visés par la Loi sur les archives (ministères et sociétés d'État, réseau municipal, de l'enseignement et de la santé). Ce guide apportait un cadre, des normes et procédures à caractère juridique, technologique et archivistique dans le but d'assurer la durabilité, l'authenticité et l'intégrité des documents numérisés. Je suis heureux d'avoir compté parmi les trois coauteurs de ce guide.

Grâce à une préparation adéquate, le passage informatique à l'an 2000 s'est bien effectué à l'Université de Montréal comme ailleurs, faisant tomber les craintes d'un «bogue de l'an 2000 ». Cette fiabilité du numérique a marqué des points et du même coup, la confiance en cette technologie s'est accrue, avec discernement.

Comme d'autres États, le Québec a ensuite adopté la Loi concernant le cadre juridique des technologies de l'information (2001). Cette loi avance la neutralité technologique dans la preuve d'intégrité des documents, et le Code civil sera ainsi amendé. Désormais, pour préserver l'intégrité d'un document, il n'est plus nécessaire que son support soit inaltérable. II faut plutôt s'assurer et pouvoir démontrer que le document n'a pas été altéré erronément ou frauduleusement. Ainsi, bien gérés, les serveurs reliés aux systèmes appropriés peuvent jouer le rôle de dépôts institutionnels de documents et archives sous forme numérique, de manière à en préserver l'authenticité et l'intégrité.

\section{LA TÂCHE DE DIRECTEUR DES ARCHIVES DE L'UNIVERSITÉ DE MONTRÉAL}

Plusieurs expériences, dont les suivantes, pour n'en nommer que quelques-unes, m'ont à la fois marqué et motivé de façon particulière à promouvoir et apporter les solutions nécessaires aux défis quotidiens de directeur des Archives de I'UdeM.

\subsection{Expériences marquantes}

Le nouveau doyen d'une grande faculté entre en fonction et cherche en vain des repères parmi les dossiers laissés par son prédécesseur. Aucun plan de classification disponible n'a été suivi, aucun ordre de classification 
n'est apparent. Le doyen erre littéralement parmi les classeurs à tiroirs et les dossiers rangés de façon arbitraire et aléatoire. Du côté des dossiers informatiques hérités dans l'un et l'autre poste de travail, la recherche est aussi pénible. Le nouveau doyen, heureusement ami des Archives et partisan d'une classification reconnaissable, me convoque à son bureau. Ne voulant plus jamais se retrouver en pareille situation, il devient un promoteur de la mise en place du Système officiel de classification des documents de I'Université (SOC) et du suivi de l'application de ce plan aux documents actifs. En outre, le doyen signale l'importance pour lui de voir apparaître sur les murs de ses locaux des illustrations de personnalités et d'activités historiques de la Faculté, faisant appel aux archives historiques. Ce doyen devient un exemple à donner et à suivre; son intérêt doit être cultivé, de multiples façons.

Un cadre académique nous révèle la disparition d'importants documents à caractère interfacultaire dans le domaine de la santé. Les documents furent produits par un comité ponctuel dont les membres travaillaient chacun avec son ordinateur portable. Une fois le travail terminé, les membres du comité se sont dispersés, chacun s'imaginant que «l'autre » était responsable de l'exemplaire principal des documents définitifs requis pour l'année académique suivante. L'été passe, la nouvelle année académique est sur le point de démarrer, et les documents requis sont introuvables au moment nécessaire. Cela a pour conséquence des délais et le report des activités de la mission principale de l'institution. De tels incidents sont rarement ébruités. Et pour chaque cas ainsi révélé, combien sont passés sous silence, pour ne pas affecter la prestation de I'institution? Leçon retenue: il est important d'utiliser un serveur agissant comme dépôt institutionnel de documents.

Par surcroît, il y eut cette rencontre avec le secrétaire général de I'Université et un nouveau directeur général des technologies de l'information et de la communication (DGTIC). Très amical et motivé face à son mandat, ce nouveau directeur était cependant convaincu, à priori, que les documents informatiques n'avaient rien à voir avec les Archives, et que le Service des archives n'avaient pas d'affaire dans les documents informatiques, les archives étant essentiellement le papier! Heureusement, pour le convaincre du contraire, il a suffi d'un examen, ensemble, des articles les plus pertinents et les plus évidents de la Loi sur les archives et de la Loi concernant le cadre juridique des technologies de l'information. Une fois bien informé du regard et des efforts des Archives à l'endroit des 
documents informatiques, ce nouveau directeur est devenu un allié et un supporteur indéfectible, ainsi qu'un partenaire exigeant en matière de modernisation des outils technologiques, des services et des responsabilités des Archives en termes de soutien aux clients. C'est d'ailleurs grâce à la très étroite collaboration de la DGTIC et aux représentations conjointes des Archives/Secrétariat général/DGTIC auprès de la haute direction de I'Université que les Archives ont pu effectuer concrètement le passage vers la gestion intégrée des documents numériques et analogiques (GID).

Un des symptômes de l'urgence d'agir avec la GID fut I'offre de la part de différentes unités, de transférer ou verser aux Archives des documents en format numérique seulement, à prendre ou à laisser. Ces unités n'imprimaient plus certains types de documents devant être conservés à plus long terme au-delà de leur phase active. Toutes les unités de l'Université furent encouragées à informer les Archives des problèmes éprouvés en gestion de documents. Le sous-texte message était le suivant: "Si vous avez des problèmes ou des difficultés avec la gestion de documents, c'est la «faute » des Archives, ce n'est pas la vôtre, et c'est la responsabilité des Archives d'apporter la solution avec vous ». Les nombreux appels à l'aide sont devenus une source de stress, certes, mais en même temps un fabuleux levier pour la promotion des solutions archivistiques et l'obtention d'appuis à cet effet. Les responsabilités des Archives furent étendues, en donnant la priorité à la gestion rigoureuse des séries de documents essentiels à la mission de l'Université.

\subsection{Représentation interne}

Cette croissance de la popularité et de l'attention portée à la gestion de documents et des archives est favorisée par la qualité des interventions et par le travail constant de représentation fait par la direction du Service des Archives auprès de groupes et d'instances stratégiques de I'Université. Bien sûr, il y a la Commission des archives, dont la forte composition et le rôle capital furent expliqués par d'autres conférenciers avant moi. II y a aussi le Comité des secrétaires de facultés et le Regroupement des directeurs de services généraux (Finances, Ressources humaines, Immeubles, TI, Communications, Registraire, Archives, etc.). Importantes aussi sont les représentations concertées auprès de la hiérarchie, la haute direction et les décideurs lorsqu'il faut affirmer avec clarté et concision, démontrer, proposer et convaincre du bien-fondé des projets du Service des Archives. 


\section{RÉALISATIONS D'ÉQUIPE}

En tant que directeur mon rôle a surtout consisté à motiver les membres de l'équipe des Archives: donner des orientations et fixer des objectifs avec eux, les appuyer et, ma foi, "leur nuire le moins possible» dans la poursuite de ces objectifs. Toujours veiller à ce que les cibles soient optimales, réalistes, conductrices de reconnaissance, de satisfaction mutuelle, sans oublier le plaisir dérivé.

Parmi les réalisations d'équipe ayant ponctué ces années:

- le SOC2, refonte du Système officiel de classification des documents de l'Université;

- la mise à jour du calendrier de conservation sur la base du nouveau Recueil de la Conférence des recteurs et des principaux des universités du Québec (CREPUQ);

- I'établissement du programme de formation en gestion de documents pour le personnel de l'Université;

- recherche, acquisition et introduction d'un logiciel institutionnel de GID;

- nouveau site internet;

- mise en place d'un atelier de numérisation sérielle de masse;

- mise à jour de la politique sur la gestion de documents et les archives de l'Université;

- le tout associé au projet baptisé Hippocampe (nom de l'organe cérébral de la mémoire et petit animal fascinant) dont parleront d'autres conférenciers au cours de la journée;

- présence régulière dans le journal Forum;

- collaboration au $125^{\mathrm{e}}$ anniversaire de I'UdeM : riche historique sur le Web, exposition des documents fondateurs, etc. ;

- collection Baby classée bien culturel historique;

- acquisition du fonds Hans Selye, médecin, professeur et chercheur découvreur du syndrome du stress. Installation en hommage à Selye à la Faculté de médecine et au Centre d'éducation physique et des sports de I'UdeM (CEPSUM); 
- solution au problème du retard de traitement d'une quantité très excessive de dossiers étudiants à l'échelle de l'Université;

- étude d'opportunité de la votation électronique à l'UdeM.

La liste des réalisations pourrait s'allonger et s'est prolongée sous la direction subséquente de Diane Baillargeon qui nous en parlera tout à I'heure en même temps qu'elle abordera les défis et projets d'avenir de la DGDA. Et puisque le temps passe, je voudrais maintenant conclure pour ma part.

\section{CONCLUSION}

Le constat n'est pas nouveau, mais toujours d'actualité. Des études ont démontré que dans les organisations:

- $80 \%$ de l'information documentaire numérique est sous forme «non structurée » (c'est-à-dire issue de logiciels tels que les texteurs, par exemple, en saisie libre, sans structure classificatoire optimale ni gestion inhérente du cycle de vie);

- plus de $60 \%$ des décisions se prennent par courriers électroniques et fichiers joints sans gestion adéquate non plus.

La majorité grandissante de ces informations ne sera jamais imprimée sur papier. Mais d'autre part, le papier est toujours présent et il sert encore. Sous forme analogique ou numérique, les archives sont une source essentielle d'information et d'inspiration. La modernisation technologique continue de la gestion de documents et archives est vitale pour préserver cette «mémoire d'utilité courante et historique». 